\title{
Article@Virology
}

\section{A glance on anti-viral strategy target Coronavirus life cycle}

Yue Zhang ${ }^{I \#}$, Huijie Chen ${ }^{2 \#}$, Nicole Pirozzi ${ }^{3}$, Yingying Cong ${ }^{3 *}$

1. College of Veterinary Medicine, Northeast Agricultural University, Harbin 150030, P.R.China

2. College of Biological and Pharmaceutical Engineering, Jilin Agricultural Science and Technology

University, Jilin 132101, P.R. China

3. Department of Biomedical Sciences of Cells \& Systems, University Medical Center Groningen,

University of Groningen, A. Deusinglaan 1, 9713 AV Groningen, The Netherlands

\begin{abstract}
Coronavirus (CoV), is a single-stranded positive-sense RNA virus, which is characterized by a coronal-shaped spinous process on its surface and is the largest known RNA virus. Recently, the new outbreak of CoV was first found in Wuhan, China at the end of 2019, however, the infection is worldwide and causing high pathogenicity and mortality rates, especially in the aged population. Unfortunately, there is no available strategy to control the infection or treat patients. By reviewing the life cycle of $\mathrm{CoV}$ infection in host cells, including: virion attachment and entry, genome transcription and replication, and virion assembly and release, we focused on the role of viral proteins in the viral life cycle and summarized how their function could be targeted for the prevention/therapy of CoV. Thus, this information would pave the way to precisely design the antiviral drug component/vaccine against CoVs.
\end{abstract}

Copyright@2012-2025 Published by Hong Kong Institute of Biologicals Standardization Limited. All rights reserved.

Article history: Submitted: 29/01/2020; Revised: 04/02/2020; Accepted: 12/02/2020

DOI:10.21092/jav.v9i1.87

Key Words: Coronavirus, Anti-viral strategy, Review

\# These authors contributed equally to this work.

* Corresponding author, PhD. , Major in Pathogeny biology

E-mail: y.cong@umcg.nl 


\section{Characterization of Coronaviruses}

Coronaviridae is subdivided into four groups based on phylogenetic clustering: alpha-, beta-, gamma- and delta- Cornavirus $(\mathrm{CoV})^{[1,2]}$. Members of this virus family infect the mammalian respiratory and gastrointestinal tracts by mechanisms that are not completely understood.

Recently, a new CoV, Severe Acute Respiratory Syndrome Coronavirus 2 (SARS-COV-2), first identified in Wuhan (China) in December 2019 ${ }^{[3,4]}$, caused $\mathrm{CoV}$ disease 2019 (COVID-19). Pandemics of SARS-COV-2 infections have been serious threats to global health, causing significant morbidity and mortality. The common signs of infection include fever, fatigue and subsequent respiratory symptoms, such as cough and pneumonia ${ }^{[5]}$. As of March 9, 2020, 80,905 infectious cases and 3,123 deaths in China (data came from National Health Commission of China) have been reported. Like Severe Acute Respiratory Syndrome Coronavirus (SARS-CoV) and Middle East Respiratory Syndrome (MERS$-\mathrm{CoV})$, SARS-COV-2 infects the population include the elderly people that could lead to a life-threatening respiratory failure and acute respiratory distress syndrome. Interestingly, SARS-COV-2 shares $79.5 \%$ of its genome with SARS-CoV, however, the homology with Bat-COV RaTG13 is as high as $96 \%$, so it is speculated that the virus originated from bats ${ }^{[6]}$, but it is not clear through which intermediate hosts the virus infects humans.

$\mathrm{CoV}$ is a kind of RNA virus with envelope and linear positive sense single strands. The 5 'end of the virus genome has a methylated cap structure, and the 3 ' end has a poly (A) tail [7]. The $\mathrm{CoV}$ genome is about $27-32 \mathrm{~kb}$ and typically encodes four structural proteins: the spike protein $(\mathrm{S})$, envelope protein $(\mathrm{E})$, membrane protein $(\mathrm{M})$, and nucleocapsid protein $(\mathrm{N})$; some $\mathrm{CoVs}$ additionally have a haemagglutinin-esterase protein (HE) ${ }^{[8,9]}$. The CoV genome also encodes for 15 or 16 nonstructural proteins (nsp1 to nsp15/16), which are synthesized in the host cell and assemble into the replicase-transcriptase complexes $(\mathrm{RTCs})^{[9]}$. Papain-like protease (PLPro) activity and the chymotrypsin-like main protease (Mpro/3CLpro) activity are encoded by nsp3 and nsp5, respectively, whereas nsp12 encodes the critical RNA-dependent RNA polymerase $(\operatorname{RdRp})^{[10]}$.

The World Health Organization (WHO) has warned that COVID-19 poses a "grave threat" to the world. There is currently no effective vaccine or anti-viral drug to target SARS-COV-2. In this paper, we review the role of $\mathrm{CoV}$ proteins in viral life cycle, including virion attachment and entry, genome transcription and replication, and virion assembly and release, this will contribute to our understanding of the virus prevention and therapy. 


\section{Life cycle of $\mathrm{CoV}$}

The CoV replication cycle in the host cell is generally divided into several steps, including virion attachment and entry, viral genome transcription and replication, translation of structural proteins, and virion assembly and release.

\subsection{Attachment and Entry}

The whole CoV replication cycle occurs in the cytoplasm of the host cell. The initial adhesion of virus to host cells is mediated by the interaction between the spike $(\mathrm{S})$ protein and its cell receptor (Figure 1, step 1). In most CoVs, the $\mathrm{S}$ protein is cleaved by host proteases into two functional subunits of roughly the same size, S1 and S2 domains. The N-terminal of $\mathrm{S} 1$ domain makes up the globular head of the $\mathrm{S}$ protein in the virion, whereas the S2 domain constitutes the stem of the $\mathrm{S}$ protein ${ }^{[11]}$. The receptor-binding domain (RBD) is located mostly in the S1 domain of $\mathrm{CoV} S$ proteins [12-14]. Interestingly, it has been reported that the RBD of some CoVs (including MERS-CoV, Human Coronavirus 229E (HCOV-229E), Transmissible Gastroenteritis Virus (TGEV), Porcine Epidemic Diarrhea Virus (PEDV) and Porcine Respiratory Coronavirus (PRCV)) is located at the C-terminal of S1 domain ${ }^{[15-19]}$. Alternatively, the RBD of Mouse Hepatitis Virus (MHV) and also PEDV are located at the N-terminal of S1 [20, 21]. The difference in location of the RBD in PEDV could be due to the different PEDV strains used. Viruses with different tissue tropism have their own specific receptors, as shown in Table 1, for most of the CoVs including HCoV-229E, TGEV, Porcine Delta-coronavirus (PDCOV), Feline Infectious Peritonitis Virus(FIPV), and Canine coronavirus (CCOV), amino-peptidase $\mathrm{N}$ (APN) is the functional receptor, ${ }^{[22-26]}$; angiotensin converting enzyme 2 (ACE2) is the functional receptor for SARS-COV-2 、 SARS-CoV and Human Coronavirus NL63 (HCoV-NL63) [27-29]; dipeptidyl peptidase 4 (DPP4) is the functional receptor of MERS-CoV [30]; carcinoembryonic antigen associated cell adhesion molecule 1 (CEACAM1) is the functional receptor of $\mathrm{MHV}$ [31] and 9-O-acetylated sialic acid is the functional receptor of Human Coronavirus OC43 (HCoV-OC43) and Human Coronavirus HKU1 (HCoV-HKU1) ${ }^{[11]}$. Additionally, to promote the $\mathrm{CoVs}$ entering, cells may need other co-factors, e.g. C-type lectins DC-SIGN and/or L-SIGN (collectively referred to as DC/L-SIGN) is/are alternative receptors for most $\mathrm{CoVs}$ (including SARS-CoV, HCoV-229E, IBV and FCoV), lectin promotes the entry of the virus into host cells by binding to glycosylated $\mathrm{S}$ proteins [29, 32-34]. Interestingly, Han et al. have identified seven glycosylation sites on the SARS-S protein that are critical for DC/L-SIGN-mediated viral entry by site-directed mutagenesis analysis. Importantly, the seven glycosylation sites located at amino acid positions 109, 118, 119, 


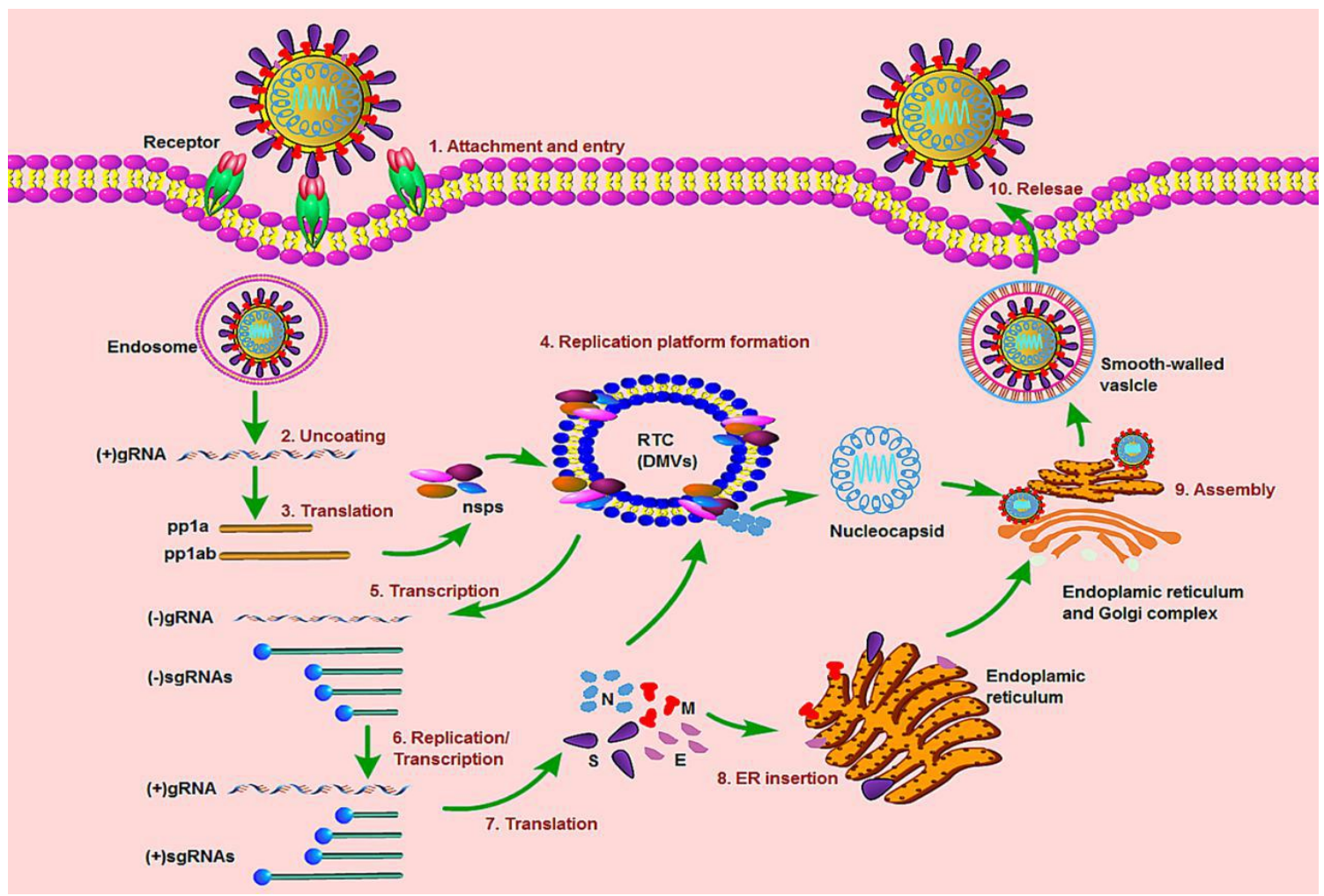

Figure 1: Life cycle of CoVs infection in host cells

Infection starts with the attachment of CoVs to the host cells and binds to specific receptors (step 1), which induces endocytosis. Membrane fusion typically occurs in the endosomes, after uncoating, releasing the viral genomic RNA (gRNA) to the cytoplasm (step 2). The genomic RNA (gRNA) serves as the template for translation of polyproteins ppla and pplab (step 3) are cleaved to form nonstructural proteins (nsps). Nsps induce the rearrangement of cellular membrane to form double-membrane vesicles (DMVs), where the viral replication transcription complexes (RTCs) are anchored (step 4). The RTCs first engage in minus-strand RNA synthesis to produce both single strand full-length and subgenomic (sg) minus-strand RNAs (step 5). Subsequently, they use sg mRNAs as templates for the production of the gRNA and plus-strand sg mRNAs required to express the structural protein genes (step 6). These sg RNAs encode S, E, and M structural proteins and then inserted in the endoplasmic reticulum (ER) (step 7 and 8). The $\mathrm{N}$ protein is expressed in the ribosome in the cytoplasm and forms a dimer around RTC, and then binds to the RNA formed in RTC to form a nucleocapsid. Virion assembly takes place in the ER-Golgi intermediate complex (ERGIC) (step 9), and mature virion are released in smooth-walled vesicles via the secretory pathway (step 10). 
158, 227, 589, and 699, which are distinct from residues of the ACE2-binding domain (amino acids 318 to 510) ${ }^{[35]}$.

After binding to the receptor, the conformation of the viral $\mathrm{S}$ protein changes. This is especially true for the S2 domain, which contains the fusion peptide (FP), two heptad repeat regions (HR1 and HR2), the transmembrane (TM) domain and the cytosolic tail ${ }^{[36]}$. The FP is further embedded into the host cell membrane, then three HR1s are folded to form a barrel-shaped central structure, and another three HR2s are wrapped in reverse parallel around the HR1s to form a six-helix bundle (6-HB) ${ }^{[27-28]}$. The conformation change of this structure could shorten the distance between the viral membrane and the cell membrane, then the two membranes are brought close enough to facilitate membrane fusion ${ }^{[37]}$.

\subsection{Genome transcription and replication}

Following the binding of the viral particle to cell receptor, the subsequent cell entry is through the membrane fusion in endosomes

Table 1. Summary of the cell receptor for CoVs

\begin{tabular}{|c|c|c|c|c|}
\hline Virus type & Virus & Receptor & co-factors & References \\
\hline \multirow{9}{*}{$\alpha-\mathrm{CoV}$} & $\mathrm{HCoV}-229 \mathrm{E}$ & $\mathrm{APN}$ & DC/L-SIGN & $(22,29)$ \\
\hline & HCoV-NL63 & ACE2 & Sialic acid & $(112)$ \\
\hline & FIPV & APN & DC/L-SIGN & $(25,34)$ \\
\hline & PRCV & APN & & (16) \\
\hline & TGEV & APN & Sialic acid & $(23,113)$ \\
\hline & PEDV & Unknown & Sialic acid & (19) \\
\hline & SADS-CoV & Unknown & & (114) \\
\hline & $\mathrm{CCoV}$ & $\mathrm{APN}$ & & (26) \\
\hline & $\mathrm{HCoV}-\mathrm{OC} 43$ & Sialic acid & & (11) \\
\hline \multirow{8}{*}{$\beta-\mathrm{CoV}$} & HCoV-HKU1 & Sialic acid & & (11) \\
\hline & $\mathrm{SARS}_{-} \mathrm{COV}_{\mathrm{V}}$ & ACE2 & cathepsin L & (28) \\
\hline & & DC/L-SIGN & HAT & $(115,116)$ \\
\hline & SARS-CoV-2 & ACE2 & & $(6,27)$ \\
\hline & MERS-CoV & DPP4 & & (30) \\
\hline & MHV & CEACAM1 & & $(31,117)$ \\
\hline & $\mathrm{BCOV}$ & $\begin{array}{c}\mathrm{N} \text {-acetyl-9-O-a } \\
\text { cetylneuramini } \\
\text { c acid }\end{array}$ & & (118) \\
\hline & PHEV & $\begin{array}{c}\mathrm{N} \text {-acetyl-9-O-a } \\
\text { cetylneuramini } \\
\text { c acid }\end{array}$ & $\begin{array}{l}\text { Neural cell } \\
\text { adhesion } \\
\text { molecule } \\
\text { (NCAM) }\end{array}$ & $(119,120)$ \\
\hline$\gamma-\mathrm{CoV}$ & IBV & Sialic acid & $\begin{array}{c}\text { Poly-LacNAc } \\
\text { DC-SIGN }\end{array}$ & $\begin{array}{c}(121) \\
(33,122)\end{array}$ \\
\hline $\mathrm{g}-\mathrm{CoV}$ & PDCoV & APN & & (24) \\
\hline
\end{tabular}


upon endocytosis. After genomic RNA uncoating from the nucleocapsid (Figure 1, step 2), CoV replication begins with the translation of ORF $1 \mathrm{a}$ and $1 \mathrm{~b}$ into poly-proteins ppla and pplab (Figure 1, step 3). Here, the downstream of ORF1b is translated through the ribosomal frameshifting mechanism, in which a translating ribosome shifts one nucleotide in the direction, from the ORF1a reading frame into ORF1b reading frame. This repositioning is enabled by two RNA elements-a 5'-UUUAAAC-3' heptanucleotide slippery sequence and RNA pseudoknot structure ${ }^{[38]}$. These polyproteins self-process into 11 and 16 individual nsp, respectively, many of the nsps then assemble into the RTC (Figure 1, step 4) [39]. Hagemeijer et al. have shown that NSP2 could recruit RTCs more effectively than other nsp to create an environment suitable for RNA synthesis ${ }^{[40]}$. Among all the $\mathrm{CoV}$ nsps, nsp3, nsp4 and nsp6 are known to contain transmembrane (TM) domains to facilitate their insertion into the endoplasmic reticulum (ER) membrane ${ }^{[41]}$, Angelini et al. further showed that these three nsps could reorganize the ER membrane to form double membrane vesicles (DMVs) in SARS-COV

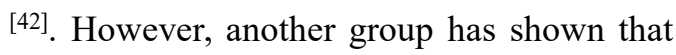
NSP3/NSP4 is essential, not nsp6, for the formation of DMV in MERS-CoV ${ }^{[43]}$. Then DMVs are integrated into the reticular network of the ER membrane, also known as the convoluted membrane (CM), and then RTC is anchored on the curled membrane as a platform for virus replication (Figure 1, step 4).

With the assembly of the replicase-polymerase, the full-length positive strand of genomic RNA is initially transcribed to form a full-length negative-strand template for the synthesis of new genomic RNAs (gRNA) (Figure 1, step 5) and overlapping subgenomic negative-strand templates (Figure 1, step 6). Several heterologous nuclear ribonucleoprotein (hnRNP) family members (including hnRNPA1, PTB and SYN-CRYP) have been found to be essential for efficient RNA replication [44, 45]. Other RNA-binding proteins have also been suggested to play a role in $\mathrm{CoV}$ replication, e.g. m-aconitase and poly-A-binding protein (PABP), DDX1, PCBP1/2 and zinc finger CCHC-type and RNA-binding motif 1 (MADP1) ${ }^{[46-48]}$. These subgenomic mRNAs are then transcribed and translated to produce the structural proteins and accessory proteins, e.g. the SARS-CoV genome codes for eight accessory proteins, namely ORFs $3 \mathrm{a}, 3 \mathrm{~b}, 6,7 \mathrm{a}, 7 \mathrm{~b}, 8 \mathrm{a}, 8 \mathrm{~b}$, and $9 \mathrm{~b}{ }^{[41]}$ and MERS-COV codes for five accessory proteins, namely ORFs 3, 4a, 4b, 5 and $8 b$ [49]. Subsequently, the transmembrane structural proteins (including S, HE, M, and E) and some membrane-associated accessory proteins are then translated and synthesized on rough ER membranes (Figure 1, step 7 and 8), whereas the $\mathrm{N}$ protein is translated by the cytosolic free ribosomes (Figure 1, step 7) ${ }^{[50]}$. After that, the self-binding of $\mathrm{N}$ protein 
is carried out to form an oligomer, which is recruited to the replication transcription site, where the DMVs and the CMs are located through the interaction with nsp3 [51, 52]. In addition, Cong et al. indicated that the recruitment of $\mathrm{N}$ protein to nsp3 through the residues F128, Y129, Y130A, T133, L240, V241, and L242 of MHV N protein, is critical for optimal viral RNA synthesis ${ }^{[53]}$. Moreover, nsp3 could induce a conformational change in the C-terminal domain (CTD) of $\mathrm{N}$ protein, revealing that its disulfide bond combined with genomic RNA to form nucleocapsid ${ }^{[54,55]}$. Specifically, within the N-terminal domain (NTD) of SARS-CoV-N, positively charged lysine and arginine residues have been proposed to bind a 32 nucleotide stem-loop structure located at the CTD of the SARS-CoV RNA genome [56]. Chen et al. reported that the CTD of SARS-CoV N protein, spanning residues 248-365 (NP248-365), is capable of binding to single-stranded RNA (ssRNA), single-stranded DNA (ssDNA) and double-stranded DNA (dsDNA) with greater affinities than the NTD [57]. Consistently, another study of X-ray crystal structures has shown that the CTD residues 248-280 of $\mathrm{N}$ protein could form a positively charged patch ${ }^{[51]}$, and the oligonucleotide binding is possibly based on non-specific charge interactions between the positively charged protein and the negatively charged nucleic acid backbone, which helps to form a large helical nucleocapsid core.

\subsection{Virion assembly and release}

The newly translated viral structural proteins, S, E, and $M$ are synthesized on the ER membrane [58], and move along the secretory pathway to the ER-Golgi intermediate compartment (ERGIC) (Figure

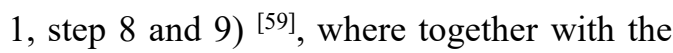
nucleocapsid, they assemble into virion by inducing the invagination and luminal pinching off of the limiting membrane that leads to the formation of intralumenal mature virions ${ }^{[60]}$. The $\mathrm{M}$ protein is the main core shell component and directs most protein-protein interactions (e.g M-M、M-E、 $M-N 、 M-S)$, which are required for the assembly of these CoV viral particles [61, 62]. A previous study has shown that a 16 amino acid domain (aa 237-252) on the CTD of TGEV-M protein binds directly to $\mathrm{N}$ protein via an ionic interaction, leading to specific genome encapsidation in the budding viral particle [63]. Interestingly, He et al. identified a stretch of amino acids (168-208) in SARS-CoV N protein that may be critical for $\mathrm{N}-\mathrm{M}$ protein interaction. Moreover, this stretch spans the linker region (LKR; residues 60-219) and dimerization domain in the CTD of $\mathrm{N}$ protein, suggesting that this domain might be critical in maintaining the correct $\mathrm{N}$ protein conformation for both self-association and N-M protein interaction [64]. Another study in MHV suggested that the $M$ protein-nucleocapsid interaction is initiated by a direct binding of $\mathrm{M}$ protein to gRNA [65], however, it still needs to be 
further clarified in other CoVs. Moreover, the $M$ protein is not sufficient for virion formation, as virus-like particles (VLPs) cannot be formed by $\mathrm{M}$ protein expression alone [66]. In contrast, when $M$ protein is co-expressed with E protein, VLPs are formed, suggesting that these two proteins function together to produce $\mathrm{CoV}$ envelopes [66]. N protein enhances VLP formation, indicating that encapsidated genomes enhance viral envelopment at the ERGIC [61].

Following assembly, virions are transported to the extracellular milieu in exocytic vesicles and released by secretion (Figure 1, step 10) [60, 67, 68]. It remains unknown whether virus use the traditional pathway for transport of large cargo from Golgi or whether virus has devised a separate, unique pathway for its own exit. Over the course of $\mathrm{CoV}$ infections, the excess $S$ proteins that do not get incorporated into virions, $\mathrm{S}$ protein traffics to cell surface where it mediates cell-cell fusion between infected cells and adjacent, uninfected cells $[67,69,70]$. This leads to the formation of giant, multinucleated cells, which allows virus to spread within an infected tissue/organism without being detected or neutralized by immune system. Various host factors have been implicated in the assembly and release of coronavirus. In particular, interactions between the cytoskeleton and structural proteins seems to be essential. Rudiger et al. have shown that the interaction between tubulin and the cytosolic domain of S protein in HCoV-229E, HCoV-NL63, and TGEV are crucial for successful assembly and release of infectious viral particles from the host cell ${ }^{[68]}$.

\section{The prevention and therapy of $\mathrm{CoV}$ target viral life cycle}

The CoVs cause economically devastating diseases of livestock, poultry, and laboratory rodents. Most CoVs infect epithelial cells in respiratory and/or enteric tracts, causing epizootics of respiratory diseases and/or gastroenteritis with short incubation periods (2-14 days), such as those found in SARS-CoV, MERS-CoV and SARS-CoV-2. Here, we will review the possible preventive strategy and therapy through the role of viral proteins in $\mathrm{CoV}$ life cycle.

\subsection{Antiviral strategy target viral $\mathrm{S}$ protein}

The membrane-anchored glycoprotein, S, is a major immunogenic antigen and is essential for the interaction between the virus and the host cell receptor. As noted previously, S1 protein is mainly involved in the recognition and binding between $\mathrm{CoV}$ and host cell receptors, while $\mathrm{S} 2$ protein mainly promotes the fusion of $\mathrm{CoV}$ and membranes ${ }^{[11]}$. Therefore, the development of a serotype vaccine or an antibody with S-glycoprotein as a target is undoubtedly a good therapeutic strategy. At present, most reports focus on the development of vaccines or antibodies against $\mathrm{S}$ protein or $\mathrm{S} 1$ protein. For instance, since MERS-CoV infection is initiated by the binding of $\mathrm{CoV} S$ glycoprotein and DPP4 [30], MERS-CoV 
S/S1 glycoprotein antibodies have been studied extensively in recent years. Jia et al. reported that the construction of recombinant rare serotype chimpanzee adenovirus expressing full-length of MERS-CoV $\mathrm{S}$ protein could induce anti-MERS-CoV antibody and $\mathrm{T}$ cell response in mice, indicating that the induction of $\mathrm{S}$ protein consanguineous antibody could well inhibit MERS-CoV infection [71]. In another study, Liu et al. developed a recombinant MERS-CoV that swapped the CoV S gene to vesicular stomatitis virus (VSV) G gene (VSV- $\Delta$ G-MERS), immunization with a single intramuscular or nasal dose of VSV- $\Delta$ G-MERS induced the neutralization of antibodies and $\mathrm{T}$ cell responses in rhesus monkeys, suggesting that the VSV- $\Delta \mathrm{G}$ -MERS chimeric vaccine has the potential to be a candidate vaccine for rapid response to MERS-CoV [72]. Moreover, the monoclonal antibody 7D10 of MERS-CoV S protein that binds to the NTD of $\mathrm{S}$ protein can also effectively inhibit the entry of MERS-CoV into cells ${ }^{[73]}$. Besides that, a complete human neutralizing antibody MCA1, was isolated from a MERS-CoV infected survivor that could recognize the RBD of MERS-CoV S protein and further block the interaction between S protein and DPP4 in common marmosets ${ }^{[74]}$. Thus, the MCA1 could be a potential alternative therapy for the treatment of human MERS-CoV infection, nevertheless, further clinical evaluation is needed.
Similar to MERS-CoV, the preparation of antibodies or the development of drugs through $\mathrm{S}$ protein targets could also be helpful to inhibit SARS-CoV infection. For instance, by targeting a specific epitope on the $\mathrm{S} 1$ subunit RBD to inhibit the binding of $\mathrm{CoV}$ to cell receptors, $\mathrm{Zhu}$ et al have confirmed that RBD of SARS-CoV S1 proteins could induce strong neutralizing antibodies and long-term protective immunity in rabbits and mice, which could completely protect the immunized mice from SARS-CoV infection ${ }^{[75]}$. Urtica dioica agglutinin (UDA) has been shown that it could inhibit SARS-CoV infection by targeting the early stages of the virus replication cycle (adsorption or penetration). Kumaki et al. further found that the UDA could neutralize the infectivity of the SARS-CoV by binding to $S$ protein (76).

In addition, Chang et al. expressed the $\mathrm{S}$ protein (S-Bac) or S1 protein (S1-Bac) of another porcine $\mathrm{CoV}$, PEDV, in baculovirus, and immunized mice and piglets with S-Bac or S1-Bac showed significant increase of the specific IgG level of S antibody, however, the S-Bac group produced better PEDV specific neutralizing antibodies, compared to S1-Bac immunized piglets [77]. The reason could be that both S1 and S2 domains of PEDV have viral effective neutralizing epitopes and the protective immune effect of the full length $\mathrm{S}$ protein is better than the $\mathrm{S} 1$ domain alone ${ }^{[78]}$. Likewise, Ke et al. have shown that the expression of PEDV $\mathrm{S}$ 
protein in highly attenuated recombinant VSV could effectively induce pig-specific immunity through muscle immunization, which is expected to be a good platform for the preparation of PEDV subunit vaccine ${ }^{[79]}$. Moreover, Pascuak-Iglesias et al. have engineered an attenuated virus based on the TGEV genome, expressing a chimeric $\mathrm{S}$ protein of PEDV (rTGEV-RS-S PEDV), which provided protection against PEDV in animals and could be a promising candidate vaccine for virulent PEDV vaccine ${ }^{[80]}$.

Since $\mathrm{CoV}$ infection is initiated by transmembrane $\mathrm{S}$ glycoprotein, the development of corresponding vaccines and drugs targeting the $\mathrm{S}$ protein would be an effective strategy against a certain $\mathrm{CoV}$ through the early stage of viral infection.

\subsection{Antivirus strategy target other structural} proteins of $\mathrm{CoV}$

Although the $\mathrm{S}$ protein plays a key role in virus life cycle, the antibodies and drugs designed on $\mathrm{S}$ protein might lose the protective effect due to the genetic mutation of $\mathrm{S}$ protein during the CoVs revolution. Compared to the $\mathrm{S}$ protein, other $\mathrm{CoV}$ structural proteins are more conserved, therefore, the design of antibodies or drugs on those structural proteins could be a mild developmental strategy for anti-CoVs in the future.

$\mathrm{N}$ protein, the main component of the nucleocapsid core of $\mathrm{CoV}$, is mainly involved in RNA synthesis and virion assembly in the process of virus replication
$[53,81]$. Since N protein of CoVs is located at the center of the virion and is rarely mutated, it could be beneficial to the develop corresponding $\mathrm{N}$ protein serotype antibodies or drugs [81, 82]. At present, the antibody vaccines and key targets of $\mathrm{N}$ protein have also been widely studied. For example, Cong et al. reported that the $\mathrm{N}$ protein is recruited to RTCs through the binding with nsp3, and then stimulate the MHV RNA synthesis [53]. With this discovery, screening of drug compounds or antibodies that specifically affect N-nsp3 binding are intensely needed and such drugs will further block viral RNA synthesis. On the other hand, there are more serotype antibodies against $\mathrm{N}$ protein, for instance, immunization of mice with the recombinant Modified Vaccinia virus with MERS-CoV N protein (MVA-MERS-N) could induce specific $\mathrm{T}$ cellular immune response and have a significant inhibitory effect on MERS-CoV infection [83]. Moreover, immunization of mice with recombinant baculovirus with bat SARS-like $\mathrm{CoV} \mathrm{N}$ protein (SL-CoV) could induce humoral and cellular immune responses [84], showing that the secretion level of IFN- $\gamma$ increased significantly and the immune response of CD4 $\mathrm{T}$ cells was significantly enhanced. Since the calreticulin (CRT) associated antigen DNA vaccine has been wildly used and could significantly enhance the presentation of major histocompatibility complex class I to CD8 (+) T cells $[85,86]$, Kim et al created an effective DNA vaccine 
of CRT linked to a SARS-CoV N protein and could induce a strong humoral immune response in mice [87]. Likewise, there is another DNA vaccine with pcDN-fn vector to express SARS-CoV N protein, which upon intramuscular injection in mice could induce both $\mathrm{N}$ protein-specific antibody and specific cytotoxic $\mathrm{T}$ lymphocyte (CTL) activity ${ }^{[88]}$. Luo et al. constructed an attenuated S. enterica serovar Typhi strain stably expressing SARS-CoV $\mathrm{N}$ protein by integrating the $\mathrm{N}$ gene into the pilV gene, which was under the control of the type IVB pilus operon promoter in S. enterica serovar Typhi. Immunization of this recombinant strain through intranasal administration, caused the mice to produce significantly specific immunoglobulins $\mathrm{G}$ ( $\mathrm{IgG}$ ), IgG2a and secretory IgA ${ }^{[89]}$. The anti-viral inhibitors that target $\mathrm{CoV} N$ protein have been studied as well. Lin et al. identified and developed a new HCoV-OC43 $\mathrm{N}$ protein inhibitor, N-(6-oxo-5,6-dihydrophenanthri-din-2-yl)(N,N-dimethylamino) acetamide hydrochloride (PJ34) through virtual screening, which inhibits the viral replication through affecting the binding affinity of the $\mathrm{N}$ protein to RNA ${ }^{[90]}$.

Similar to the $\mathrm{N}$ protein, the $\mathrm{M}$ protein of CoVs is also a highly conserved viral structural protein, which is essential for virion assembly [91, 92]. Therefore, it is also worth it to develop vaccine or drugs based on $\mathrm{M}$ protein to target CoVs. As of now, Wang et al. identified two B cell epitopes from SARS-CoV $M$ protein through bioinformatics analysis and constructed a multi-epitope DNA vaccine. Intramuscular immunization in mice induces specific challenge and long-term memory of the humoral immune response [93]. Likewise, another group found the intramuscular immunization in mice with the expression of SARS-CoV M protein in pcDNA3.1 vector could also induce significant cellular and humoral immune responses [94]. Moreover, Pang et al. showed that the rabbit antisera to SARS M protein also had high neutralizing titer against SARS-CoV infection in rabbit, indicating that $\mathrm{M}$ protein as an immunogen could induce strong SARS-CoV specific neutralizing antibody response ${ }^{[95]}$.

Although the development of vaccines or drugs targeting the highly conserved $\mathrm{CoV} N$ or $\mathrm{M}$ proteins has achieved certain results and might be a development trend of anti-CoVs in the future, it still needs further clinical evaluation.

\subsection{Anti-CoV strategy target nsps and} accessory proteins of $\mathrm{CoV}$

As noted before, the nsps of $\mathrm{CoV}$ are critical for viral replication and transcription ${ }^{\text {[96] }}$. Antiviral drugs have been widely studied by targeting domains of PLpro, 3CLpro and RdRp in nsp3, nsp5 and nsp12 [97]. Interestingly, some inhibitors could affect not only a specific $\mathrm{CoV}$, for example, the 6-mercaptopurine and 6-thioguanine, the inhibitors for the PLpro in both SARS-CoV and MERS-CoV, resulting the blocking of 
viral infection efficiently ${ }^{[98] .}$ The lopinavir-ritonavir, as a 3CLpro inhibitor, has anti-CoV activity in MERS-CoV infected primates and SARS-infected patients ${ }^{[99,100]}$. Moreover, Warren et al developed a novel broad-spectrum nucleoside analogue BCX4430, which could inhibit the infection of numerous viruses in human cells, including CoVs, by inhibiting RdRp [101]. Likewise, the remdesivir (RDV, GS-5734) is an RdRp inhibitor, which has been shown to decrease the viral replication in MERS-CoV and SARS-CoV [102, 103], as well as SARS-COV-2, and is currently being usedto treat infected patients in the United States and China ${ }^{[104,105] \text {. }}$

Since nsp 15 is an endonuclease for $\mathrm{CoVs}$ and is essential to evade the dsRNA sensor, nsp 15 could be considered as a candidate for the anti-CoV vaccine [106]. Deng et al. found the viral infection of two independent mutant nsp15 MHV strains in bone marrow-derived macrophages were poorly replicated and greatly alleviated the disease in mice. This may be due to the fact that the mutation of nsp15 will affect the endonuclease of CoVs, thus affecting the replication of $\mathrm{CoVs}{ }^{[107]}$. Therefore regulating the stability and activity of CoV nsp15 could also be a strategy to design live attenuated vaccine. Besides that, nsp16 of CoVs encode conserved 2'O methyltransferase (2'-MTase), which is an important domain of RNA replication. Menachery et al. mutated the conserved motif of nsp16 in MERS-CoV through reverse genetic assay and found an attenuated effect in infected human airway cells, indicating that nsp 16 could also be a target for live attenuated vaccine ${ }^{[108]}$.

The accessory proteins of CoVs, whose size is only a small part of the viral genome (less than 10kb), are almost not necessary for viral replication, nevertheless, some of them have been proved to play an important role in the pathogenesis of the virus ${ }^{[109]}$. Few studies have focused on the development of $\mathrm{CoV}$ accessory proteins protein as a vaccine target. For instance, the Oligoadenylate synthase (OAS)-ribonuclease L (RNase L) pathway is activated when the viral double-stranded RNA is sensed, and activated RNase $\mathrm{L}$ cleave the viral single-stranded RNA, to prevent further virus replication and transmission [110], Comar et al. showed the MERS-CoV NS4b accessory protein could antagonize the effect of OAS-RNaseL to promote viral replication [111], indicating NS4b might be a potential target for therapeutic intervention of MERS-CoV.

Since the nsps and accessory proteins of CoVs are also essential for viral replication, it is worthwhile to consider the development of antiviral drugs/antibodies targeting nsps/ accessory proteins.

\section{Conclusions}

In the past two decades, the pandemics of $\mathrm{CoV}$ infections have been serious threats to global health, causing significant morbidity and mortality. In particular, SARS-CoV, 
MERS-CoV and SARS-COV-2, have caused minor to major outbreaks of viral pneumonia worldwide despite advances in their treatments. Consequently, it is crucial to study in-depth the CoV life cycle to better understand how they interact with cellular pathways and thus potentially develop more effective therapies.

Most of the drug component/vaccine targeted $\mathrm{CoV} \mathrm{S}$ protein to inhibit the viral entry step, however, $S$ protein varies a lot in different strains of a $\mathrm{CoV}$, and it is a challenge to guarantee that a constructed drug component/vaccine targeting $\mathrm{S}$ protein would be effective to all strains. Further investigations are therefore needed to investigate anti-viral strategy that target the conserved viral proteins, like the $\mathrm{M}$ protein, the $\mathrm{N}$ protein or nsps. Another drawback of several studies cited in this review is that the development of such drug components /vaccines are still studied in vitro or with pre-limited data in mice. Although that knowledge could be key to designing the antiviral drug component/vaccine, they can have adverse effects in clinic and the following evaluation of those strategies still need to be further investigated.

\section{Acknowledgments}

Y.C. was supported by a Chinese Scholarship Council PhD fellowship (grant CSC 201406610008). The authors apologize in advance to those authors whose contributions have been omitted due to lack of space and felt that their work was one of the highlights.

\section{Reference}

[1]. Fung TS, Liu DX. 2019. Human Coronavirus: Host-Pathogen Interaction[J]. Annu Rev Microbiol,2019, 73:529-557.

[2]. Siddell S G, Anderson R, Cavanagh D, et al. Coronaviridae[J]. Intervirology, 1983, 20(4): 181-189.

[3]. Huang C, Wang Y, Li X, et al.Clinical features of patients infected with 2019 novel coronavirus in Wuhan, China[J]. Lancet 2020.,(20)30183-5.

[4]. Chan JF, Kok KH, Zhu Z, et al. 2020. Genomic characterization of the 2019 novel human-pathogenic coronavirus isolated from a patient with atypical pneumonia after visiting Wuhan[J]. Emerg Microbes Infect,2020, 9:221-236.

[5]. Huang C, Wang Y, Li X, et al. Clinical features of patients infected with 2019 novel coronavirus in Wuhan, China[J]. The lancet, 2020, 395(10223): 497-506.

[6]. Zhou P, Yang X L, Wang X G, et al. Discovery of a novel coronavirus associated with the recent pneumonia outbreak in humans and its potential bat origin[J]. BioRxiv, 2020.

[7]. Perlman S, Netland J. Coronaviruses post-SARS: update on replication and pathogenesis[J]. Nat Rev Microbiol,2009, 7:439-50. 
[8]. de Wit E, van Doremalen N, Falzarano D, Munster VJ. SARS and MERS: recent insights into emerging coronaviruses $[\mathrm{J}]$. Nat Rev Microbiol,2016, 14:523-34.

[9]. Chen Y, Liu Q, Guo D. Emerging coronaviruses: genome structure, replication, and pathogenesis[J]. Journal of medical virology, 2020, 92(4): 418-423.

[10]. Xu X, Liu Y, Weiss S, et al. Molecular model of SARS coronavirus polymerase: implications for biochemical functions and drug design[J]. Nucleic Acids Res,2003, 31:7117-30.

[11]. Huang X, Dong W, Milewska A, et al. 2015. Human Coronavirus HKU1 Spike Protein Uses O-Acetylated Sialic Acid as an Attachment Receptor Determinant and Employs Hemagglutinin-Esterase Protein as a Receptor-Destroying Enzyme[J]. J Virol,2015, 89:7202-13.

[12]. Cheng PK, Wong DA, Tong LK, et al. Viral shedding patterns of coronavirus in patients with probable severe acute respiratory syndrome[J]. Lancet,2004, 363:1699-700.

[13]. Graham RL, Baric RS. Recombination, reservoirs, and the modular spike: mechanisms of coronavirus cross-species transmission[J]. J Virol,2010, 84:3134-46.

[14]. Wan Y, Shang J, Graham R, et al. Receptor recognition by the novel coronavirus from Wuhan: an analysis based on decade-long structural studies of SARS coronavirus[J]. Journal of virology, 2020, 94(7).
[15]. Reguera J, Santiago C, Mudgal G, et al. Structural bases of coronavirus attachment to host aminopeptidase $\mathrm{N}$ and its inhibition by neutralizing antibodies[J]. PLoS pathogens, 2012, 8(8).

[16]. Reguera J, Ordono D, Santiago C, et al. Antigenic modules in the N-terminal S1 region of the transmissible gastroenteritis virus spike protein[J]. J Gen Virol,2011, 92:1117-26.

[17]. Li Z, Tomlinson A C A, Wong A H M, et al. The human coronavirus HCoV-229E S-protein structure and receptor binding[J]. Elife, 2019, 8: e51230.

[18]. Delmas B, Gelfi J, Kut E, et al. Determinants essential for the transmissible gastroenteritis virus-receptor interaction reside within a domain of aminopeptidase- $\mathrm{N}$ that is distinct from the enzymatic site[J]. J Virol, 1994, 68:5216-24.

[19]. Deng F, Ye G, Liu Q, et al. Identification and Comparison of Receptor Binding Characteristics of the Spike Protein of Two Porcine Epidemic Diarrhea Virus Strains[J]. Viruses,2016, 8:55.

[20]. Kubo H, Yamada YK, Taguchi F. Localization of neutralizing epitopes and the receptor-binding site within the amino-terminal 330 amino acids of the murine coronavirus spike protein [J]. J Virol ,1994, 68:5403-10. 
[21]. Kim S H, Cho B H, Lee K Y, et al. N-terminal domain of the spike protein of porcine epidemic diarrhea virus as a new candidate molecule for a mucosal vaccine[J]. Immune network, 2018, 18(3).

[22]. Yeager CL, Ashmun RA, Williams RK, et al. 1992. Human aminopeptidase $\mathrm{N}$ is a receptor for human coronavirus 229E[J]. Nature $357: 420-2$.

[23]. Weingartl HM, Derbyshire JB. Cellular receptors for transmissible gastroenteritis virus on porcine enterocytes[J]. Adv Exp Med Biol,1995, 380:325-9.

[24]. Wang B, Liu Y, Ji C M, et al. Porcine deltacoronavirus engages the transmissible gastroenteritis virus functional receptor porcine aminopeptidase $\mathrm{N}$ for infectious cellular entry[J]. Journal of virology, 2018, 92(12): e00318-18.

[25]. Tresnan DB, Levis R, Holmes KV. Feline aminopeptidase $\mathrm{N}$ serves as a receptor for feline, canine, porcine, and human coronaviruses in serogroup I[J]. J Virol ,1996, 70:8669-74.

[26]. Benbacer L, Kut E, Besnardeau L, et al. Interspecies aminopeptidase- $\mathrm{N}$ chimeras reveal species-specific receptor recognition by canine coronavirus, feline infectious peritonitis virus, and transmissible gastroen-teritis virus[J]. J Virol,1997, 71:734-7.

[27]. Wu Y. Compensation of ACE2 function for possible clinical management of 2019-nCoVinduced acute lung injury[J]. Virologica Sinica, 2020: 1-3.
[28]. Bosch BJ, Bartelink W, Rottier PJ. 2008. Cathepsin L functionally cleaves the severe acute respiratory syndrome coronavirus class I fusion protein upstream of rather than adjacent to the fusion peptide[J]. J Virol,2008, 82:8887-90.

[29]. Jeffers SA, Hemmila EM, Holmes KV. Human coronavirus 229E can use CD209L (L-SIGN) to enter cells[J]. Adv Exp Med Biol ,2006, 581:265-9.

[30]. Raj VS, Mou H, Smits SL, et al. Dipeptidyl peptidase 4 is a functional receptor for the emerging human coronavirus-EMC[J]. Nature,2013, 495:251-4.

[31]. Nedellec P, Dveksler GS, Daniels E, et al. Bgp2, a new member of the carcinoembryonic antigen-related gene family, encodes an alternative receptor for mouse hepatitis viruses. J Virol,1994, 68:4525-37.

[32]. Jeffers SA, Tusell SM, Gillim-Ross L, et al. CD209L (L-SIGN) is a receptor for severe acute respiratory syndrome coronavirus[J]. Proc Natl Acad Sci U S A,2004, 101:15748 -53 .

[33]. Zhang Y, Buckles E, Whittaker GR. Expression of the C-type lectins DC-SIGN or L-SIGN alters host cell susceptibility for the avian coronavirus, infectious bronchitis virus[J]. Vet Microbiol,2012, 157:285-93.

[34]. Regan AD, Whittaker GR. Utilization of DC-SIGN for entry of feline coronaviruses into host cells[J]. J Virol,2008, 82:11992-6. 
[35]. Han DP, Lohani M, Cho MW. Specific asparagine-linked glycosylation sites are critical for DC-SIGN- and L-SIGN-mediated severe acute respiratory syndrome coronavirus entry[J]. J Virol,2007, 81:12029-39.

[36]. Sergel TA, McGinnes LW, Morrison TG. 2001. Mutations in the fusion peptide and adjacent heptad repeat inhibit folding or activity of the Newcastle disease virus fusion protein[J]. J Virol,2001, 75:7934-43.

[37]. Bosch BJ, van der Zee R, de Haan CA, et al. The coronavirus spike protein is a class I virus fusion protein: structural and functional characterization of the fusion core complex[J]. J Virol,2003, 77:8801-11.

[38]. Lim Y X, Ng Y L, Tam J P, et al. Human coronaviruses: a review of virus-host interactions[J]. Diseases, 2016, 4(3): 26.

[39]. Ziebuhr J, Snijder EJ, Gorbalenya AE. Virus-encoded proteinases and proteolytic processing in the Nidovirales[J]. J Gen Virol,2000, 81:853-79.

[40]. Hagemeijer MC, Verheije MH, Ulasli M, et al. Dynamics of coronavirus replication-transcription complexes[J]. J Virol,2010, 84:2134-49.

[41]. Knoops K, Kikkert M, Worm SH, et al. SARS-coronavirus replication is supported by a reticulovesicular network of modified endoplasmic reticulum[J]. PLoS Biol,2006, 6:e226.

[42]. Angelini M M, Akhlaghpour M, Neuman B W, et al. Severe acute respiratory syndrome coronavirus nonstructural proteins 3,4 , and 6 induce double-membrane vesicles[J]. MBio,
2013, 4(4): e00524-13.

[43]. Oudshoorn D, Rijs K, Limpens R W A L, et al. Expression and cleavage of Middle East respiratory syndrome coronavirus nsp3-4 polyprotein induce the formation of double-membrane vesicles that mimic those associated with coronaviral RNA replication[J]. MBio, 2017, 8(6): e01658-17.

[44]. Luo H, Chen Q, Chen J, et al. The nucleocapsid protein of SARS coronavirus has a high binding affinity to the human cellular heterogeneous nuclear ribonucleoprotein A1[J]. FEBS Lett,2005, 579:2623-8.

[45]. Li Z, Zeng W, Ye S, et al. Cellular hnRNP A1 Interacts with Nucleocapsid Protein of Porcine Epidemic Diarrhea Virus and Impairs Viral Replication[J]. Viruses, 2018, 10(3): 127..

[46]. Nanda SK, Leibowitz JL. Mitochondrial aconitase binds to the 3 ' untranslated region of the mouse hepatitis virus genome[J]. J Virol ,2001,75:3352-62.

[47]. Wu CH, Chen PJ, Yeh SH. Nucleocapsid phosphorylation and RNA helicase DDX1 recruitment enables coronavirus transition from discontinuous to continuous transcription [J]. Cell Host Microbe, 2014,16: 462-72.

[48]. Tan YW, Hong W, Liu DX. Binding of the 5 -untranslated region of coronavirus RNA to zinc finger CCHC-type and RNA-binding motif 1 enhances viral replication and transcription[J]. Nucleic Acids Res,2012, 40:5065-77.

[49]. Yang Y, Zhang L, Geng H, et al. The structural and accessory proteins $\mathrm{M}, \mathrm{ORF} 4 \mathrm{a}, \mathrm{ORF} 4 \mathrm{~b}$, and ORF 5 of Middle East respiratory 
syndrome coronavirus (MERS-CoV) are potent interferon antagonists[J]. Protein Cell,2013, 4:951-61.

[50]. Masters PS. The molecular biology of coronaviruses[J]. Advances in virus research ,2006,66:193-292.

[51]. Chen C Y, Chang C, Chang Y W, et al. Structure of the SARS coronavirus nucleocapsid protein RNA-binding dimerization domain suggests a mechanism for helical packaging of viral RNA[J]. Journal of molecular biology, 2007, 368(4): 1075-1086.

[52]. Yu IM, Gustafson CL, et al. Recombinant severe acute respiratory syndrome (SARS) coronavirus nucleocapsid protein forms a dimer through its C-terminal domain[J]. J Biol Chem,2005, 280:23280-6.

[53]. Cong Y, Ulasli M, Schepers H, et al. Nucleocapsid protein recruitment to replication-transcription complexes plays a crucial role in coronaviral life cycle $[\mathrm{J}]$. Journal of virology, 2020, 94(4)..

[54]. Hurst K R, Ye R, Goebel S J, et al. An interaction between the nucleocapsid protein and a component of the replicase-transcriptase complex is crucial for the infectivity of coronavirus genomic $\mathrm{RNA}[\mathrm{J}]$. Journal of virology, 2010, 84(19): 10276-10288.

[55]. McBride R, Van Zyl M, Fielding B C. The coronavirus nucleocapsid is a multifunctional protein[J]. Viruses, 2014, 6(8): 2991-3018.
[56]. Huang Q, Yu L, Petros A M, et al. Structure of the N-terminal RNA-binding domain of the SARS CoV nucleocapsid protein[J]. Biochemistry, 2004, 43(20): 6059-6063.

[57]. Takeda M, Chang CK, Ikeya T et al. Solution structure of the c-terminal dimerization domain of SARS coronavirus nucleocapsid protein solved by the SAIL-NMR method[J]. J Mol Biol ,2008,380:608-22.

[58]. Krijnse-Locker J, Ericsson M, Rottier P J, et al. Characterization of the budding compartment of mouse hepatitis virus: evidence that transport from the RER to the Golgi complex requires only one vesicular transport $\operatorname{step}[\mathrm{J}]$. The Journal of Cell Biology, 1994, 124(1): 55-70.

[59]. Tooze J, Tooze S, Warren G. Replication of coronavirus MHV-A59 in sac- cells: determination of the first site of budding of progeny virions[J]. Eur J Cell Biol,1984, 33:281-93.

[60]. de Haan CA, Rottier PJ. Molecular interactions in the assembly of coronaviruses [J]. Adv Virus Res,2005, 64:165-230.

[61]. Siu YL, Teoh KT, Lo J, et al. The M, E, and N structural proteins of the severe acute respiratory syndrome coronavirus are required for efficient assembly, trafficking, and release of virus-like particles[J]. J Virol,2008, 82: 11318-30. 
[62]. Nguyen V P, Hogue B G. Protein interactions during coronavirus assembly[J]. Journal of virology, 1997, 71(12): 9278-9284.

[63]. Escors D, Ortego J, Laude H, et al. The membrane $\mathrm{M}$ protein carboxy terminus binds to transmissible gastroenteritis coronavirus core and contributes to core stability[J]. Journal of virology, 2001, 75(3): 1312-1324.

[64]. He R, Leeson A, Ballantine $M$, et al. Characterization of protein-protein interactions between the nucleocapsid protein and membrane protein of the SARS coronavirus[J]. Virus research, 2004, 105(2): 121-125.

[65]. Narayanan K, Maeda A, Maeda J, et al. Characterization of the coronavirus $\mathrm{M}$ protein and nucleocapsid interaction in infected cells[J]. Journal of virology, 2000, 74(17): 8127-8134.

[66]. BOS E C W, LUYTJES W, VAN DER MEULEN $\mathrm{H}$, et al. The production of recombinant infectious DI-particles of a murine coronavirus in the absence of helper virus[J]. Virology, 1996, 218(1): 52-60.

[67]. Fehr AR, Perlman S. Coronaviruses: an overview of their replication and pathogenesis[J]. Methods Mol Biol, 2015, 1282:1-23.

[68]. Rüdiger A T, Mayrhofer P, Ma-Lauer Y, et al. Tubulins interact with porcine and human $\mathrm{S}$ proteins of the genus Alphacoronavirus and support successful assembly and release of infectious viral particles[J]. Virology, 2016, 497: 185-197.
[69]. Gelhaus S, Thaa B, Eschke K, et al. Palmitoylation of the Alphacoronavirus TGEV spike protein $\mathrm{S}$ is essential for incorporation into virus-like particles but dispensable for S-M interaction[J]. Virology, 2014, 464: 397-405..

[70]. Ye R, Montalto-Morrison C, Masters PS. Genetic analysis of determinants for spike glycoprotein assembly into murine coronavirus virions: distinct roles for charge-rich and cysteine-rich regions of the endodomain[J]. J Virol,2004, 78:9904-17.

[71]. Jia W, Channappanavar R, Zhang C, et al. Single intranasal immunization with chimpanzee adenovirus-based vaccine induces sustained and protective immunity against MERS-CoV infection[J]. Emerg Microbes Infect ,2019, 8:760-772.

[72]. Liu R, Wang J, Shao Y, et al. 2018. A recombinant VSV-vectored MERS-CoV vaccine induces neutralizing antibody and $\mathrm{T}$ cell responses in rhesus monkeys after single dose immunization[J]. Antiviral Res, 2018, 150:30-38.

[73]. Zhou H, Chen Y, Zhang S, et al. Structural definition of a neutralization epitope on the $\mathrm{N}$-terminal domain of MERS-CoV spike glycoprotein[J]. Nature communications, 2019, 10(1): 1-13.

[74]. Chen Z, Bao L, Chen C, et al. Human neutralizing monoclonal antibody inhibition of middle east respiratory syndrome coronavirus replication in the common marmoset[J]. The Journal of infectious diseases, 2017, 215(12): $1807-1815$. 
[75]. Zhu X, Liu Q, Du L, et al. Receptor-binding domain as a target for developing SARS vaccines[J]. Journal of thoracic disease, 2013, 5(Suppl 2): S142.

[76]. Kumaki Y, Wandersee MK, Smith AJ, et al. Inhibition of severe acute respiratory syndrome coronavirus replication in a lethal SARS-CoV BALB/c mouse model by stinging nettle lectin, Urtica dioica agglutinin[J]. Antiviral Res,2011, 90:22-32.

[77]. Chang C Y, Hsu W T, Chao Y C, et al. Display of porcine epidemic diarrhea virus spike protein on baculovirus to improve immunogenicity and protective efficacy[J]. Viruses, 2018, 10(7): 346.

[78]. Li C, Li W, de Esesarte E L, et al. Cell attachment domains of the porcine epidemic diarrhea virus spike protein are key targets of neutralizing antibodies[J]. Journal of virology, 2017, 91(12): e00273-17.

[79]. Ke Y, Yu D, Zhang F, et al. Recombinant vesicular stomatitis virus expressing the spike protein of genotype $2 \mathrm{~b}$ porcine epidemic diarrhea virus: A platform for vaccine development against emerging epidemic isolates[J]. Virology, 2019, 533:77-85.

[80]. Pascual-Iglesias A, Sanchez C M, Penzes Z, et al. Recombinant Chimeric Transmissible Gastroenteritis Virus (TGEV)—Porcine Epidemic Diarrhea Virus (PEDV) Virus Provides Protection against Virulent PEDV[J]. Viruses, 2019, 11(8): 682.

[81]. McCormick C, Khaperskyy DA. Translation inhibition and stress granules in the antiviral immune response[J]. Nat Rev Immunol,
2017,17:647-660.

[82]. Emmott E, Munday D, Bickerton E, et al. The cellular interactome of the coronavirus infectious bronchitis virus nucleocapsid protein and functional implications for virus biology[J]. Journal of virology, 2013, 87(17): 9486-9500.

[83]. Veit S, Jany S, Fux R, et al. CD8+ T cells responding to the middle east respiratory syndrome coronavirus nucleocapsid protein delivered by vaccinia virus MVA in mice[J]. Viruses, 2018, 10(12): 718.

[84]. Bai B, Lu X, Meng J, et al. Vaccination of mice with recombinant baculovirus expressing spike or nucleocapsid protein of SARS-like coronavirus generates humoral and cellular immune responses[J]. Molecular immunology, 2008, 45(4): 868-875..

[85]. Kim D, Hoory T, Monie A, et al. Enhancement of DNA vaccine potency through coadministration of CIITA DNA with DNA vaccines via gene gun[J]. J Immunol,2008, 180:7019-27.

[86]. Cheng WF, Hung CF, Chen CA, et al. Characterization of DNA vaccines encoding the domains of calreticulin for their ability to elicit tumor-specific immunity and antiangiogenesis[J]. Vaccine,2005, 23:3864 -74 .

[87]. Kim TW, Lee JH, Hung CF, et al. Generation and characterization of DNA vaccines targeting the nucleocapsid protein of severe acute respiratory syndrome coronavirus[J]. J Virol,2004, 78:4638-45. 
[88]. Zhu M S, Pan Y, Chen H Q, et al. Induction of SARS-nucleoprotein-specific immune response by use of DNA vaccine[J]. Immunology letters, 2004, 92(3): 237-243..

[89]. Luo F, Feng Y, Liu M, et al. Type IVB pilus operon promoter controlling expression of the severe acute respiratory syndrome-associated coronavirus nucleocapsid gene in Salmonella enterica Serovar Typhi elicits full immune response by intranasal vaccination $[\mathrm{J}]$. Clin. Vaccine Immunol., 2007, 14(8): 990-997.

[90]. Lin SY, Liu CL, Chang YM, et al. Structural basis for the identification of the N-terminal domain of coronavirus nucleocapsid protein as an antiviral target[J]. J Med Chem,2014, 57:2247-57.

[91]. Neuman BW, Kiss G, Kunding AH, et al. A structural analysis of $\mathrm{M}$ protein in coronavirus assembly and morphology[J]. J Struct Biol,2011, 174:11-22.

[92]. Ujike M, Taguchi F. Incorporation of spike and membrane glycoproteins into coronavirus virions[J]. Viruses, 2015, 7:1700-25.

[93]. Wang $\mathrm{X}, \mathrm{Xu} \mathrm{W}$, Tong $\mathrm{D}$, et al. A chimeric multi-epitope DNA vaccine elicited specific antibody response against severe acute respiratory syndrome-associated coronavirus which attenuated the virulence of SARS-CoV in vitro[J]. Immunology letters, 2008, 119(1-2): 71-77..

[94]. Wang Z, Yuan Z, Matsumoto M, et al. Immune responses with DNA vaccines encoded different gene fragments of severe acute respiratory syndrome coronavirus in BALB/c mice $[\mathrm{J}]$. Biochem Biophys Res
Commun,2005, 327:130-5.

[95]. Pang H, Liu Y, Han X, et al. Protective humoral responses to severe acute respiratory syndrome-associated coronavirus: implica-tions for the design of an effective protein-based vaccine $[\mathrm{J}]$. Journal of general virology, 2004, 85(10): 3109-3113.

[96]. Brian DA, Baric RS. Coronavirus genome structure and replication[J]. Curr Top Microbiol Immunol,2005, 287:1-30.

[97]. Zumla A, Chan JF, Azhar EI, et al. Coronaviruses-drug discovery and therapeutic options. Nat Rev Drug Discov,2016, 15:327-47.

[98]. Cheng KW, Cheng SC, Chen WY, et al. Thiopurine analogs and mycophenolic acid synergistically inhibit the papain-like protease of Middle East respiratory syndrome coronavirus[J]. Antiviral Res,2015, 115:9-16.

[99]. Chan KS, Lai ST, Chu CM, et al. Treatment of severe acute respiratory syndrome with lopinavir/ritonavir: a multicentre retrospective matched cohort study [J]. Hong Kong Med J,2003, 9:399-406.

[100]. Chan J F W, Yao Y, Yeung M L, et al. Treatment with lopinavir/ritonavir or interferon- $\beta 1 \mathrm{~b}$ improves outcome of MERS-CoV infection in a nonhuman primate model of common marmoset[J]. The Journal of infectious diseases, 2015, 212(12): 1904-1913.

[101]. Warren TK, Wells J, Panchal RG, et al. Protection against filovirus diseases by a novel broad-spectrum nucleoside analogue BCX4430[J]. Nature ,2014,508:402-5. 
[102]. Sheahan T P, Sims A C, Graham R L, et al. Broad-spectrum antiviral GS-5734 inhibits both epidemic and zoonotic coronaviruses[J]. Science translational medicine, 2017, 9(396). [103]. de Wit E, Feldmann F, Cronin J, et al. Prophylactic and therapeutic remdesivir (GS-5734) treatment in the rhesus macaque model of MERS-CoV infection[J]. Proceedings of the National Academy of Sciences, 2020, 117(12): 6771-6776.

[104]. Holshue M L, DeBolt C, Lindquist S, et al. First case of 2019 novel coronavirus in the United States[J]. New England Journal of Medicine, 2020.

[105]. Guan W, Ni Z, Hu Y, et al. Clinical characteristics of coronavirus disease 2019 in China[J]. New England journal of medicine, 2020, 382(18): 1708-1720.

[106]. Deng X, Baker SC. An "Old" protein with a new story: Coronavirus endoribonuclease is important for evading host antiviral defenses. Virology 2018,517:157-163.

[107]. Deng X, Hackbart M, Mettelman R C, et al. Coronavirus nonstructural protein 15 mediates evasion of dsRNA sensors and limits apoptosis in macrophages[J]. Proceedings of the National Academy of Sciences, 2017, 114(21): E4251-E4260.

[108]. Menachery V D, Gralinski L E, Mitchell H D, et al. Middle east respiratory syndrome coronavirus nonstructural protein 16 is necessary for interferon resistance and viral pathogenesis[J]. mSphere, 2017, 2(6): e00346-17.
[109]. Zhao L, Jha B K, Wu A, et al. Antagonism of the interferon-induced OAS-RNase L pathway by murine coronavirus ns 2 protein is required for virus replication and liver pathology[J]. Cell host \& microbe, 2012, 11(6): 607-616.

[110]. Choi UY, Kang JS, Hwang YS, et al. Oligoadenylate synthase-like (OASL) proteins: dual functions and associations with diseases[J]. Exp Mol Med,2015, 47:e144.

[111]. Thornbrough JM, Jha BK, Yount B, et al. Middle East Respiratory Syndrome Coronavirus NS4b Protein Inhibits Host RNase L Activation[J]. mBio,2016, 7:e00258.

[112]. Hofmann H, Pyrc K, van der Hoek L, et al. Human coronavirus NL63 employs the severe acute respiratory syndrome coronavirus receptor for cellular entry[J]. Proc Natl Acad Sci U S A,2005, 102:7988-93.

[113]. Krempl C, Schultze B, Laude H, et al. 1997. Point mutations in the $\mathrm{S}$ protein connect the sialic acid binding activity with the enteropathogenicity of transmissible gastro-enteritis coronavirus[J]. J Virol,1997, 71:3285-7.

[114]. Yang Y L, Qin P, Wang B, et al. Broad cross-species infection of cultured cells by bat HKU2-related swine acute diarrhea syndrome coronavirus and identification of its replication in murine dendritic cells in vivo highlight its potential for diverse interspecies transmission[J]. Journal of virology, 2019, 93(24). 
[115]. Li W, Moore MJ, Vasilieva N, et al. Angiotensin-converting enzyme 2 is a functional receptor for the SARS coronavirus[J]. Nature,2003, 426:450-4.

[116]. Bertram S, Glowacka I, Müller M A, et al. Cleavage and activation of the severe acute respiratory syndrome coronavirus spike protein by human airway trypsin-like protease[J]. Journal of virology, 2011, 85(24): 13363-13372.

[117]. Taguchi F, Hirai-Yuki A. Mouse hepatitis virus receptor as a determinant of the mouse susceptibility to $\mathrm{MHV}$ infection[J]. Frontiers in microbiology, 2012, 3: 68.

[118]. Schultze B, Herrler G. Bovine coronavirus uses $\mathrm{N}$-acetyl-9-O-acetylneuraminic acid as a receptor determinant to initiate the infection of cultured cells $[\mathrm{J}]$. Journal of General Virology, 1992, 73(4): 901-906.

[119]. Hulswit R J G, Lang Y, Bakkers M J G, et al. Human coronaviruses OC43 and HKU1 bind to 9-O-acetylated sialic acids via a conserved receptor-binding site in spike protein domain $\mathrm{A}[\mathrm{J}]$. Proceedings of the National Academy of Sciences, 2019, 116(7): 2681-2690.
[120]. Dong B, Gao W, Lu H, et al. A small region of porcine hemagglutinating encephalo-myelitis virus spike protein interacts with the neural cell adhesion molecule[J]. Intervirology,2015, 58:130-7.

[121]. Shahwan K, Hesse M, Mork A K, et al. Sialic acid binding properties of soluble coronavirus spike (S1) proteins: differences between infectious bronchitis virus and transmissible gastroenteritis virus[J]. Viruses, 2013, 5(8): 1924-1933.

[122]. Wickramasinghe I N A, de Vries R P, Weerts E, et al. Novel receptor specificity of avian gammacoronaviruses that cause enteritis[J]. Journal of virology, 2015, 89(17): 8783-8792.. 